\title{
Uso da testosterona no envelhecimento masculino
}

\author{
Use of testosterone in men's aging \\ Igor Tupinambá Calixto ${ }^{1 *}$, Tereza Cristina M. de Melo Prazeres ${ }^{1}$
}

\begin{abstract}
Resumo: Introdução: Com o avanço da idade, o homem pode apresentar declínio na produção de testosterona, acarretando sinais e sintomas como diminuição da libido, disfunção erétil, redução da massa muscular, acúmulo de gordura corporal, redução da densidade mineral óssea, doença endotelial e sintomas depressivos. Esta síndrome clínica e laboratorial recebe o nome de deficiência androgênica do envelhecimento masculino (DAEM) e tem forte relação com a síndrome metabólica. Objetivo: Avaliar os efeitos da terapia de reposição hormonal em pacientes hipogonádicos. Material e método: Revisão bibliográfica de artigos publicados nos últimos 10 anos. No momento em que há o aumento da expectativa de vida, o desejo de envelhecer com qualidade de vida torna-se essencial a todo ser humano. A terapia de reposição da testosterona (TRT) no homem hipogonádico, pode reduzir os sinais e sintomas mencionados acima, porém nem todos os homens estão aptos a se submeter ao tratamento. Apneia obstrutiva do sono, sintomas prostáticos severos e eritrocitose devem ser tratadas antes de iniciar a terapia. Os portadores de câncer de próstata em atividade ou prolactinoma não podem fazê-lo, e entram no quadro de contra-indicações absolutas. Conclusão: A terapia de reposição de testosterona homens com deficiência androgênica típica da terceira idade é fortemente recomendada, uma vez que ela é capaz de amenizar os sinais e sintomas do envelhecimento, melhora a síndrome metabólica, possui poucas contra-indicações e as drogas utilizadas apresentam segurança farmacológica, o que facilita o tratamento desses pacientes.
\end{abstract}

Palavras-chave: DAEM. Envelhecimento. Homem. Terapia de reposição da testosterona.

\begin{abstract}
Introduction: As men age, men may experience decline in testosterone production, leading to signs and symptoms such as low libido, erectile dysfunction, reduced muscle mass, accumulation of body fat, reduced bone mineral density, endothelial disease, and symptoms depressive disorders. This clinical and laboratory syndrome is called androgenic deficiency of male aging (DAEM) and has a strong relationship with the metabolic syndrome. Objective: To evaluate the effects of hormone replacement therapy in hypogonadal patients. Material and method: Bibliographical review of articles published in the last 10 years. At the moment when there is an increase in life expectancy, the desire to grow old with quality becomes inherent to every human being. Testosterone replacement therapy (TRT) in hypogonadal man may reduce the signs and symptoms mentioned above, but not all men are eligible to undergo treatment. Obstructive sleep apnea, severe prostatic symptoms and erythrocytosis should be treated prior to initiation of therapy. Patients with active prostate cancer or prolactinoma can not, and they fall into the category of absolute contraindications. Conclusion: Testosterone replacement therapy in men with typical androgen deficiency is highly recommended, since it is able to attenuate the signs and symptoms of aging, improves the metabolic syndrome, has few contraindications and the drugs used has pharmacological safety, which facilitates the treatment of these patients.
\end{abstract}

Keywords: DAEM. Aging. Man. Testosterone replacement therapy.

${ }^{1}$ Universidade CEUMA, Campus Renascença.

Autor correspondente*:

Igor Tupinambá Calixto

Universidade CEUMA, Campus Renascença.

Endereço: Rua Josué Montello, o1, Renascença II - CEP 65.075-120 - São Luís/MA. Fone Telefone: (98)3214-4277.

E-mail: igorcalixto_1338@hotmail.com

Rev. Investig, Bioméd. São Luís, 10(3): 227-236, 2018 


\section{Introdução}

A expectativa de vida da população brasileira cresce exponencialmente. Estima-se que em 2045, o número de pessoas com mais de 65 anos ultrapasse o número de crianças, o que aumenta as discussões entre as instituições governamentais, sociedade médicas e universidades sobre a demanda desse novo perfil populacional para que ele possa envelhecer com qualidade de vida. ${ }^{1,2}$

Durante 0 envelhecimento masculino, as concentrações séricas de testosterona tendem a diminuir devido à falência dos testículos em produzir níveis adequados de testosterona e este quadro associado às suas consequências clínicas recebe o nome de DAEM (Deficiência Androgênica do Envelhecimento Masculino). Estima-se que $20 \%$ dos idosos do mundo apresentem esta síndrome. Nos Estados Unidos, por exemplo, há uma prevalência de 4 a 5 milhões de indivíduos hipogonádicos em uma população constituída de 40 milhões de idosos. Nesse país, as vendas de soluções à base de testosterona quadruplicaram de 2000 a 2011, apesar do número de pessoas com baixos níveis laboratoriais de testosterona não ter se alterado. ${ }^{3,1}$

A terapia de reposição hormonal (TRT) visa restaurar os níveis séricos de testosterona e seus metabólitos, objetivando reverter ou amenizar o quadro clínico de hipogonadismo, além de reduzir a incidência de comorbidades como diabetes, hipertensão e síndrome metabólica. No entanto o diagnóstico de hipogonadismo é muitas das vezes negligenciado, além disso a reposição hormonal apresenta efeitos adversos que são bastante debatidos na medicina atual e que podem contraindicar o tratamento. 3,4

O tratamento do hipogonadismo
masculino não deve ser mais

subvalorizado e tem que fazer parte da abordagem clínica de todo endocrinologista e urologista, uma vez que a Brasil caminha para o envelhecimento populacional. Sendo assim o objetivo do trabalho é estudar as indicações e contra-indicações da TRT, assim como seus efeitos no organismo para que se possa indicar e acompanhar com segurança os pacientes com DAEM submetidos à mesma.

\section{Material e Método}

Trata-se de um artigo de revisão sistemática realizado nas bases de dados da PUBMED dos artigos publicados no período de 2000 a 2018, no qual foram analisados artigos já publicados sobre terapia de reposição hormonal em pacientes com DAEM.

\section{Revisão da Literatura Fisiologia da testosterona}

A maior parte das funções sexuais tanto do homem quanto da mulher, começa pela secreção pulsátil de $\mathrm{GnRH}$ pelos corpos celulares de neurônios localizados no núcleo arqueado do hipotálamo. Este peptídeo estimula a hipófise anterior a produzir dois hormônios gonadotrópicos: $\mathrm{O}$ LH (hormônio luteinizante) e o FSH (hormônio folículoestimulante). ${ }^{5}$

Diferentemente do FSH que é secretado de maneira linear, o LH segue os padrões de pulsatilidade do $\mathrm{GnRH}$ e por causa dessa estreita relação, esse hormônio também recebe o nome de hormônio liberador de $\mathrm{LH}^{5} \mathrm{O} \mathrm{FSH}$ e LH são glicoproteínas e ambas exercem seus efeitos nos tecidos-alvo dos testículos ativando 0 sistema de segundo mensageiro do monofosfato cíclico de adenosina, que por sua vez ativa sistemas enzimáticos específicos nas células-alvo. ${ }^{5}$ 
A testosterona é secretada pela células intersticiais de Leyding nos testículos apenas quando estimuladas pelo LH. Essa produção é diretamente proporcional à quantidade de $\mathrm{LH}$ disponível no organismo. ${ }^{5}$

O hipotálamo é inibido por feedback negativo promovido pela testosterona circulante. Níveis elevados desse hormônio diminuem a pulsação de $\mathrm{GnRH}$ pelo hipotálamo, enquanto baixos níveis do mesmo faz o contrário: aumenta a frequência de pulsos. ${ }^{5}$

A biossíntese da testosterona nas células de Leyding ocorre a partir do colesterol. O LH se liga aos receptores de membrana e induz a síntese de AMPc a partir do ATP. Outras enzimas transportam o colesterol para dentro da membrana interna da mitocôndria onde é convertida para pregnenolona. Este metabólito é transportado para o retículo endoplasmático liso, onde sofre diversas reações enzimáticas que culminam na liberação de testosterona para a

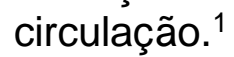

As células de Leyding são praticamente inexistentes durante a infância, mas são numerosas no homem adulto após a puberdade, época em que os testículos secretam grande quantidade de testosterona. ${ }^{5}$

Praticamente toda a testosterona (97\%) se liga fracamente à albumina plasmática ou fortemente à globulina ligadora de hormônio sexual (GLHS) e assim circula no sangue de minutos a horas. Em seguida ela é transferida para os tecidos ou degradada. Apenas a testosterona sérica livre ou ligada à albumina são biodisponíveis ${ }^{5}$. A maior parte da testosterona que se fixa nos tecidos é convertida nas próprias células do local em sua forma ativa, a dihidrotestosterona, ou é aromatizada por alguns tecidos específicos, como 0 adiposo, em estradiol. 5

Diversas são as funções da testosterona no organismo, entre elas estão fazer o posicionamento adequado dos testículos na bolsa escrotal, aumento da distribuição dos pelos corporais, aumento da espessura da pele, maior formação de proteínas e desenvolvimento muscular, retém cálcio, aumento do metabolismo basal e do número de hemácias, e hipertrofia da mucosa da laringe e seu alargamento o que da origem à voz típica do adulto. ${ }^{5}$

\section{Fisiopatologia do DAEM}

Existem várias teorias a respeito da patogênese do hipogonadismo em homens acima de 40 anos, e as principais se baseiam em 3 mecanismos: (1) diminuição do número de células de Leyding (2) secreção pulsátil do LH mais atenuada e desordenada (3) aumento da capacidade de ligação à globulina ligadora de hormônio sexual. Porém estudos mais recentes propõem uma teoria mais aceita que é a falta de resposta das células testiculares ao hormônio luteinizante. ${ }^{6}$

Hoje está mais claro que células de Leyding envelhecidas possuem déficit no número de receptores. Estudos feitos com roedores mostraram que ao aplicar $\mathrm{LH}$ em roedores velhos não há alteração nos níveis de testosterona, enquanto nos jovens os níveis aumentaram significativamente. Além disso, novos trabalhos concluíram que as células de Leyding no testículo do homem adulto, raramente se multiplicam e seu número não se altera com a idade. Apesar do avanço científico dos últimos anos, ainda faltam mais estudos para se chegar à exata patogênese da DAEM. ${ }^{1,7}$

\section{Diagnóstico}

Cada pessoa responde clinicamente diferente a este quadro porque depende da capacidade individual em manter a níveis adequados de testosterona e 
sensibilidade dos tecidos periféricos à mesma ao longo dos anos. Os sinais e sintomas clínicos do hipogonadismo são pouco específicos e por isso nem sempre reconhecidos, o que requer atenção na hora de fechar o diagnóstico. ${ }^{4}$

A Sociedade Brasileira de Urologia segue os padrões internacionais no que tange ao diagnóstico de DAEM e determina que ele é eminentemente clínico. Porém alguns autores citam uma regra que pode ajudar no raciocínio diagnóstico: homens com dosagens de testosterona total menor que $300 \mathrm{ng} / \mathrm{ml}$ são hipogonádicos, aqueles com mais de $400 \mathrm{ng} / \mathrm{ml}$ dificilmente o são, e entre 300 $400 \mathrm{ng} / \mathrm{ml}$ devem ser avaliados com base em sua clínica. ${ }^{1,8}$

Muitas das vezes o hipogonadismo não é o principal motivo da consulta devido a um cenário clínico heterogêneo. Os pacientes podem apresentar graus variados de diminuição da libido, disfunção erétil, sarcopenia e perda de massa óssea, pensamentos depressivos, perda da massa magra, aumento da massa gorda, diabetes, hipertensão, fadiga, diminuição das capacidade laboral, diminuição dos pelos corporais, fogachos e infertilidade. $O$ resultado do estudo European Male Aging Study (EMAS) realizado com 3369 homens com idade entre 40 e 79 anos mostrou que há três principais sintomas com maiores chances de prever o hipogonadismo, que são diminuição de pensamentos sexuais, disfunção erétil e ereção matinal diminuída. ${ }^{3,1}$

Levando em consideração a inespecificidade dos sintomas, o rastreamento para deficiência de testosterona através da sua dosagem sérica pode ser recomendado para homens adultos que obtiverem uma pontuação baixa e um questionário utilizado por várias instituições nacionais e internacionais, chamado ADAM (Androgen Deficiency in Aging Male). Este questionário aborda questões sexuais, psicológicas e físicas da deficiência androgênica e também é utilizado para avaliar resposta clínica ao tratamento. ${ }^{1,9}$

\section{Indicações e contraindicações}

Nem todos os pacientes que recebem o diagnóstico de hipogonadismo típico da idade podem se submeter ao tratamento. A reposição de testosterona só deve ser feita quando há sintomas e níveis séricos de testosterona total abaixo de $300 \mathrm{ng} / \mathrm{dl}$ ou $10,4 \mathrm{nmol} / \mathrm{L}$ e testosterona livre abaixo de $6,5 \mathrm{ng} / \mathrm{dL}$, dosadas em duas ocasiões diferentes, entre 7 e 11 horas da manhã. .,10 $^{3,0}$

A abordagem do paciente consiste em uma sequência ordenada de avaliação clínica e laboratorial que visa pesquisar o desejo de paternidade e possíveis contraindicações absolutas e relativas ao tratamento. As absolutas são: neoplasia de próstata não tratada com intenção curativa e prolactinomas. As relativas são: síndrome da apneia obstrutiva do sono, hiperplasia prostática com sintomas miccionais severos e poliglobulia. Nesse caso as contraindicações relativas devem ter seus sintomas controlados ou resolvidos e o paciente não deve desejar ter filhos. ${ }^{11}$

\section{Efeitos da TRT \\ Melhora da disfunção sexual}

A disfunção sexual é o sintoma mais proeminente e característico relacionado ao hipogonadismo. Entre os homens com queixas sexuais a prevalência do hipogonadismo chega a $23-36 \%$, e o uso da terapia de reposição é capaz de reverter esse quadro. Uma meta análise recente mostrou que o uso da TRT, independentemente da idade, melhorou significativamente a libido de pacientes com testosterona total abaixo de $230 \mathrm{ng} / \mathrm{dl}$ 
e disfunção erétil naqueles com testosterona abaixo de $350 \mathrm{ng} / \mathrm{dll}^{3}$

Outro estudo de Coorte feito com 999 homens, acompanhados por 3 anos, com idade entre 49-69 anos, testosterona total entre $9-10 \mathrm{~mol} / \mathrm{L}$ e diagnosticados clinicamente com hipogonadismo, mostrou que a parcela que recebeu TRT apresentou melhora constante nos seus sintomas sexuais quando comparados aos tratados com placebo. ${ }^{12}$

\section{Ganho de massa muscular}

Outra queixa comum do envelhecimento masculino que é alvo da terapia é a perda de massa muscular. Em 2017, 20 homens selecionados de um centro médico acadêmico terceirizado, com idade entre 50-70 anos diagnosticados com diabetes tipo 2, com níveis glicêmicos controlados, e testosterona livre menor que $7.3 \mathrm{nmol} / \mathrm{L}$ receberam $5 \mathrm{~g}$ de testosterona em gel para aplicar diariamente durante 24 semanas, enquanto 20 homens com o mesmo perfil receberam placebo ao longo do mesmo período e foram avaliados quanto à composição física de seus membros inferiores. Ao final do estudo, os pacientes que receberam testosterona em gel apresentaram diminuição da massa gorda e aumento da massa magra de ambas as pernas, porém seu desempenho físico não foi alterado. Já aqueles pacientes que aplicaram o placebo tiveram deterioração de sua massa magra e da função mecânica do musculo extensor do joelho, o que implica dizer que a TRT pode não só diminuir o risco de desenvolver sarcopenia como é capaz de manter o estilo de vida independente, devido à manutenção da força muscular usual, e de reduzir o tempo de reabilitação motora de pacientes idosos com baixos níveis de testosterona. ${ }^{13}$

\section{Aumento da densidade mineral óssea}

Além de manter uma massa muscular adequada, a reposição hormonal também é capaz de aumentar a densidade mineral óssea. Em 2012, 40 homens com idade entre 47-67 anos, testosterona abaixo de $320 \mathrm{ng} / \mathrm{dl}$ e síndrome metabólica receberam undecanoato de testosterona via intramuscular 4 vezes ao ano por 36 meses, e foram avaliados quanto à densidade mineral óssea do fêmur. Vinte homens de mesmo perfil clínico e que apresentavam contra-indicações ao tratamento foram utilizados como grupo controle. Após os três anos de estudo, os pacientes submetidos ao tratamento apresentaram um aumento significativo de $5 \%$ ao ano de densidade mineral óssea enquanto no grupo controle houve uma diminuição sem números estatisticamente significativos. Pode-se inferir então que a TRT é capaz de evitar o surgimento de osteoporose em homens idosos hipogonádicos. ${ }^{14}$

\section{Prevenção de síndrome metabólica e DM2}

A reposição de testosterona também é capaz de prevenir o surgimento de síndrome metabólica e diabetes tipo 2. Em homens, já há fortes evidencias que relacionam baixos níveis de testosterona, obesidade, diabetes mellitos tipo 2 (DM2) e componentes da síndrome metabólica. A obesidade é um dos maiores sintomas de pacientes hipogonádicos, e em 2015 a associação americana de endocrinologia clínica recomendou que todos os homens com IMC>30 kg/m² devem fazer o screening para hipogonadismo assim como aqueles com DM2 ou circunferência abdominal $>104 \mathrm{~cm} .{ }^{15}$

O estilo de vida sedentário e dieta hipercalórica levam ao acúmulo de gordura corporal. O aumento de tecido 
adiposo contribui para uma maior aromatização periférica da testosterona em estradiol e isso por vez diminui a secreção dos hormônios gonadotróficos pela hipófise, que consequentemente reduz a produção de testosterona pelas células de Leyding. Este baixo nível de testosterona prejudica algumas reações químicas do organismo como síntese de proteínas, ativação de células satélites musculares e lipólise, o que contribui para a manutenção do ganho de peso corporal. ${ }^{16}$

A gordura visceral é o principal fator de risco para o surgimento de resistência periférica à insulina que leva ao DM2. Uma meta-análise feita de estudos prospectivos, mostrou que homens com testosterona total maior que $15.5 \mathrm{nmol} / \mathrm{dl}$ têm $42 \%$ de chance a menos de desenvolver diabetes. Outra meta-análise realizada em 2011 expôs que a testosterona média de homens que desenvolveram DM2 era $2.08 \mathrm{nmol} / \mathrm{L}$ menor do que aqueles que não desenvolveram a doença. ${ }^{16}$

Um estudo publicado em 2014, que acompanhou 261 pacientes com idade entre 51 e 68 anos diagnosticados com hipogonadismo e disfunção erétil tratados com undecilato de testosterona de longa ação por 4 anos e 3 meses mostrou alguns resultados importantes. Após 0 tratamento, os pacientes obtiveram melhora da disfunção erétil, perderam em torno de $8 \mathrm{~kg}$, a circunferência abdominal diminuiu $8 \mathrm{~cm}$ e o índice de massa corporal antes de $31.7 \mathrm{~kg} / \mathrm{m}^{2}$ foi para $29.4 \mathrm{~kg} / \mathrm{m}^{2}$. Também houve redução do colesterol total e dos triglicerídeos, aumento do HDL e os níveis de glicose sanguínea, assim como a pressão sistólica e diastólica diminuíram. Ao final do estudo houve uma melhora significativa a qualidade de vida desses pacientes e diminuição da incidência de síndrome metabólica. ${ }^{17}$

\section{Efeitos sobre o PSA e sintomas do trato urinário baixo}

Os efeitos da terapia de reposição sobre os níveis de PSA do homem com DAEM e sintomas do trato urinário baixo ainda são inconclusivos. Estudos demonstram que não houve aumento do marcador prostático em homens diagnosticados com déficit androgênico quando submetidos ao tratamento com testosterona. Comparado ao grupo controle os níveis de PSA são semelhantes, porém os pacientes que receberam o hormônio via intramuscular apresentaram discreto aumento do marcador. ${ }^{18}$

Outros estudos afirmam que a TRT aumenta o volume prostático e os níveis de PSA de forma similar aos de homens eugonádicos envelhecidos. Dados de 7 meta-análises publicadas desde 2005 mostrou que a reposição de testosterona não está associada com a piora dos sintomas do trato urinário baixo, detecção de PSA anormal ou desenvolvimento do câncer de próstata. Porém há fortes evidências que ela pode estimular 0 crescimento prostático e agravar os sintomas miccionais em homens com câncer de próstata avançado. Apesar de não agravar os sintomas urinários ou alterar os níveis de PSA em homens hipogonádicos, a TRT é contra-indicada em pacientes com neoplasia de próstata avançada e é aconselhável tratar sintomas urinários severos em homens com HPB antes de iniciar a terapia. ${ }^{3,18}$

\section{Exacerba a apneia obstrutiva do sono}

Outra contra-indicação da TRT é a apneia obstrutiva do sono (AOS). Existe forte relação entre AOS e concentração sérica de testosterona. Baixos níveis desse hormônio significa que há uma alteração no eixo pituitário-gonadal e o distúrbio do sono mencionado é uma 
causa direta desse mecanismo prejudicado, porém quando tratado, os níveis de testosterona não retornam ao normal e se utilizar a terapia de reposição com o mesmo objetivo os sintomas da AOS são exacerbados reduzindo a saturação de oxigênio desses pacientes durante a noite. Não há evidências o suficiente que comprovem essa relação e algumas delas são inconsistentes, mas os "Guidlines" atuais mantêm como contraindicação relativa para TRT, a apneia obstrutiva do sono. Deve-se tratála, antes de submeter o paciente à reposição hormonal. ${ }^{19,20}$

\section{Infertilidade}

Outro aspecto que deve ser levado em consideração antes de iniciar a reposição hormonal em pacientes hiponádicos é o desejo de ter filhos. A testosterona exógena suprime a secreção de LH o que diminui os altos níveis de testosterona intratesticular essencial para a espermatogênese. A azoospermia nesses pacientes pode ser irreversível em $25 \%$ dos casos. Logo, pacientes que planejam de ter filhos irão iniciar 0 tratamento devem ser aconselhados a fazer a criopreservação de sêmen previamente..$^{21,10}$

\section{Aumento do hematócrito e incidência de TVP}

A poliglobulinemia também é uma contra-indicação relativa ao tratamento devido aos seus efeitos adversos. Um dos efeitos mais comuns terapia androgênica é aumentar a massa eritrocitária. Trabalhos mostram maiores de riscos de TVP em homens sob uso da TRT, principalmente naqueles com trombofilia subjacente. Também há evidências do aumento do risco de TVP nos primeiros 6 meses da terapia que diminui após esse período. Mas, outros dois grandes estudos retrospectivos mostraram aumento da incidência. Ainda faltam mais trabalhos a respeito desse tópico, mas a investigação de doenças hematológicas faz parte da abordagem dos pacientes candidatos ao tratamento e deve-se tratar a eritrocitose previamente ao tratamento. ${ }^{22,23}$

\section{Aumento do risco de eventos cardiovasculares}

Outro tópico inconclusivo é o aumento do risco cardiovascular. Três estudos e algumas meta-análises levantaram a preocupação a respeito do aumento do risco de eventos cardiovasculares (infarto agudo do miocárdio e acidente vascular encefálico) em homens tratados com TRT. Os mesmos sugerem que este aumento pode estar associado com a forma de administração intramuscular da terapia. Nova meta-análise feita que inclui 30 estudos sobre TRT e 1642 homens sugere que a reposição de testosterona em pacientes hipogonádicos e a manutenção de seus níveis na faixa fisiológica não aumentam o risco de desfechos cardiovasculares. ${ }^{24,25}$

Outro estudo ainda sobre o caso acima foi feito nos Estados Unidos da América com 3422 homens do serviço militar americano. Todos apresentavam idade entre 40-64 anos e aqueles que tinham baixos níveis de testosterona receberam TRT de 2006 a 2010. Comparados com o grupo controle ao final dos 4 anos, aquele que recebeu o tratamento teve melhora na taxa de sobrevivência a eventos cardiovasculares, menor incidência de doenças coronarianas e maior risco de desenvolver AOS. Após os estudos mencionados, pode-se observar que faltam mais trabalhos a respeito do risco de desfechos cardiovasculares nos pacientes submetidos à TRT. Por isso, 
deve-se acompanhar adequadamente 0 paciente que deseja seguir com 0 tratamento. ${ }^{20}$

\section{Acompanhamento}

Compreender o paciente e a sua correta adesão ao tratamento são essenciais para o sucesso clínico, com máximo benefício e menores taxas de efeitos adversos. O tempo para que ocorram os efeitos da TRT varia bastante. O aumento da energia, função sexual, aumento da massa magra e eritropoiese ocorrem nos primeiros três meses, enquanto os efeitos sobre a densidade mineral óssea demoram 24 meses. ${ }^{1,26}$

Após iniciar o tratamento, sugere-se avaliar a resposta clínica de 3 em 3 meses através de anamnese, exame físico (principalmente da próstata) e exames laboratoriais, como hematócrito, testosterona sérica e PSA, ajustando a dose caso ocorra resposta inadequada, testosterona em níveis subnormais e surgimento de efeitos adversos. Caso os sintomas do paciente melhorem e os exames laboratoriais estiverem dentro da normalidade nesse período, o seguimento passa a ser anual. Caso não ocorra melhora dos sintomas no período esperado deve-se considerar a interrupção do tratamento. ${ }^{10,3}$

Antes de iniciar o tratamento é importante verificar o hematócrito, se ele estiver alto, deve-se investigar a causa e tratá-la. Caso ocorra um aumento no hematócrito maior que $54 \%$ em três meses o paciente deve ser investigado para fatores de agravo como tabagismo, AOS e a TRT suspensa temporariamente ou ajustada a dose ou a forma de administração. Se 0 hematócrito permanecer alto apesar dos níveis reduzidos de testosterona considera-se a flebotomia. ${ }^{27,3}$

Em relação ao exame digital da próstata, as indicações de biópsia devem ser as mesmas utilizadas nos esquemas tradicionais de rastreamento para câncer. Elevações do PSA do estado hipogonádico para o eugonádico podem ser esperadas e elas se situam entre 0,5 $\mathrm{ng} / \mathrm{ml}$. Quando as elevações se situam entre 0,7 e $0,9 \mathrm{ng} / \mathrm{ml}$ repete-se a medida em 6 meses e caso esta se mantenha, a biopsia é indicada. Se em três meses o aumento do PSA for de $1 \mathrm{ng} / \mathrm{ml}$ encaminha-se o paciente imediatamente pra biópsia. ${ }^{1}$

Em relação à hepatotoxicidade do tratamento, as formas empregadas hoje no meio médico tem pouca toxicidade em sua maioria por serem alquiladas. Logo não há indicação do controle de provas de função hepática no homem em TRT. ${ }^{28}$

\section{Formas de apresentação}

A única forma oral utilizada com segurança é a undecanoato de testosterona que é absorvida via linfática e alcança os órgãos desejados sem ser metabolizada pelo fígado e por isso não está associada a efeitos hepáticos adversos. Porém a farmacologia individual muito variável e a ineficácia de manter os níveis de testosterona adequados a torna inadequada para tratamentos de longo tempo. ${ }^{3}$

Ésteres de testosterona de longa duração via intramuscular como enantato de testosterona e cipionato de testosterona são utilizadas há bastante tempo para tratar o hipogonadismo. A desvantagem é a necessidade de aplicação a cada uma a três semanas e as variações de testosterona sérica, que levam à instabilidade de energia, humor e libido. ${ }^{3}$

Preparações transdérmicas (adesivos, gel e solução), mantêm níveis estáveis de testosterona sérica e sua farmacocinética mimetiza as variações fisiológicas diurnas da testosterona. Pode ser interrompido seu tratamento 
imediatamente caso apresente efeitos adversos devido ao seu curto tempo de ação. Essas vantagens tornam esse tipo de apresentação a melhor modalidade de tratamento para pacientes que vão iniciar o tratamento ou que apresentem sintomas flutuantes causados por outras fórmulas. ${ }^{3}$

\section{Conclusão}

A terapia de reposição de testosterona em homens com déficit androgênico do envelhecimento masculino, apresenta mais vantagens do que desvantagens, como opçao de tratamento que 0 urologista e 0 endocrinologista podem oferecer ao seu paciente. E uma terapia que possui poucas contra-indicações absolutas e relativas que podem ser detectadas com anamnese, exame físico e laboratoriais bem direcionados. Além disso, as fórmulas de apresentação da testosterona utilizadas no meio médico tem pouca toxicidade e permitem um controle adequado dos seus níveis séricos.

Apesar de faltarem estudos a respeito de alguns efeitos adversos, o acompanhamento adequado do paciente permite que o tratamento ocorra sem intercorrências graves que não possam ser revertidas.

\section{Agradecimentos}

Agradeço primeiramente ao meu pai que sugeriu o tema deste artigo e me estimulou a pesquisar e escrever sobre, além de sanar várias dúvidas a respeito do mesmo. Também gostaria de agradecer à minha orientadora Tereza Cristina pelo seu tempo, disponibilidade e orientação.

\section{Conflito de interesse}

Os autores declaram não ter nenhum conflito de interesse.

\section{Referências}

1. Rhoden EL, Soares DF, Torres LO. Urologia Brasil, 2013; (26):297-308.

2. Wang $\mathrm{C}$, et al. Investigation, treatment, and monitoring of late-onset. hypogonadism in males: ISA, ISSAM, EAU, EAA, and ASA recommendations. Eur Urol., 2009 May; 55(5):121-30.

3. Tsametis CP, Isidori AM. Testosterone replacement therapy: for whom, when and how? Metabolism., 2018 Sep; 86:69-78.

4. Wu FCW, et al. Identification of late-onset hypogonadism in middle-aged and elderly men. N Engl J Med., 2010 Jul 8;363(2):123-35.

5. Hall JE, Guyton AC. Tratado de fisiologia médica. 2011; (80):1025-1039.

6. Buvat $J$, et al. Endocrine aspects of male sexual dysfunctions. J Sex Med., 2010 Apr;7(4):162756.

7. Midzak A, et al. Mitochondrial protein import and the genesis of steroidogenic mitochondria. Mol Cell Endocrinol., 2011 Apr 10; 336(1-2):709.

8. Wang $C$, et al. Investigation, treatment and monitoring of late-onset hypogonadism in males. Eur J Endocrinol., 2008 Nov; 159(5): 507-514.

9. Johanes CB, et al. Incidence of erectile dysfunction in men 40 to 69 years old: longitudinal results from the massachusetts male aging study. J Urol. 2000 Feb;163(2):4603.

10.Bhasin S, et al. Testosterone therapy in men with hypogonadism: an endocrine society* clinical practice guideline. J Clin Endocrinol Metab., 2018 May 1;103(5):1715-1744.

11.Rhoden EL, Morgentaler A. Risks of testosterone-replacement therapy and recommendations for monitoring. $\mathrm{N}$ Engl $\mathrm{J}$ Med., 2004;350:482-92.

12.Rosen RC et al. Quality of life and sexual function benefits of long-term testosterone treatment: longitudinal results from the registry of hypogonadism in men (RHYME). J Sex Med., 2017 Sep;14(9):1104-1115.

13.Magnussen LV, et al. Testosterone therapy preserves muscle strength and power in aging men with type 2 diabetes a randomized controlled trial. Andrology., 2017 Sep;5(5):946953. 
14.Aversa A, et al. Effects of long-acting testosterone undecanoate on boné mineral density in middle-aged men with late-onset hypogonadism and metabolic syndrome: results from a 36 months controlled study. Aging Male., 2012 Jun;15(2):96-102.

15. Hackett $\mathrm{G}$. Type 2 diabetes and testosterone therapy. World J Mens Health., 2018 Jul 17.

16. Fink J, Matsumoto M, Tamura Y. Potential application of testosterone replacement therapy as treatment for obesity and type 2 diabetes in men. Steroids., 2018 Oct;138:161-166.

17. Yassin DJ, Doros G, Hammerer PG, Yassin AA. Long-term testosterone treatment in elderly men with hypogonadism and erectile dysfunction reduces obesity parameters and improves metabolic syndrome and healthrelated quality of life. J Sex Med., 2014 Jun;11(6):1567-76.

18.Kang D, Li HJ. The Effect of Testosterone Replacement Therapy on Prostate-Specific Antigen (PSA) levels in men being treated for hypogonadism: a systematic review and metaanalysis. Medicine (Baltimore)., 2015 Jan;94(3):e410.

19.Kim SD, Cho KS. Obstructive Sleep Apnea and Testosterone Deficiency. World J Mens Health., 2018 May 16.

20.Cole AP, et al. Impact of testosterone replacement therapy on thromboembolism, heart disease and obstructive sleep apnea in men. BJU Int., 2018 May;121(5):811-818.

21.Page ST, et al. Intratesticular Androgens and Spermatogenesis During Severe Gonadotropin Suppression Induced by Male Hormonal Contraceptive Treatment. J Androl., 2007 SepOct;28(5):734-41.

22.Gluek CJ, Wang P. Testosterone therapy, thrombosis, thrombophilia, cardiovascular events. Metabolism., 2014 Aug;63(8):989-94.

23. Martinez $\mathrm{C}$, et al. Testosterone treatment and risk of venous thromboembolism: population based case-control study. BMJ., 2016;355:i5968.

24.Xu L, Freeman G, Cowling BJ, Schooling CM. Testosterone therapy and cardiovascular events among men: a systematic review and meta-analysis of placebo-controlled randomized trials. BMC Med., 2013 Apr 18;11:108.

25. Haddad et al. Testosterone and cardiovascular risk in men: a systematic review and meta- analysis of randomized placebo-controlled trials. Mayo Clin Proc., 2007 Jan;82(1):29-39.

26.Snyder PJ, et al. Effects of testosterone replacement in hypogonadal men. $\mathrm{J}$ Clin Endocrinol Metab., 2000 Aug;85(8):2670-7.

27.Lunenfeld B, Saad F, Hoels CE. ISA, ISSAM and $E A U$ recommendations for the investigation, treatment and monitoring of lateonset hypogonadism in males: scientific background and rationale. Aging Male. 2005 Jun;8(2):59-74.

28. Halmenschlager G, Rhoden EL, Riedner CE. Calculated free testosterone and radioimmunoassay free testosterone as a predictor of subnormal levels of total testosterone. Int Urol Nephrol., 2012 Jun;44(3):673-81. 